\title{
Accelerometer data treatment for adolescents: Fitting a piece of the puzzle
}

\author{
Melody Smith ${ }^{\mathrm{a}, *}$, Steve Taylor ${ }^{\mathrm{b}}$, Leon Iusitini ${ }^{\mathrm{c}}$, Tom Stewart ${ }^{\mathrm{d}}$, Fa'asisila Savila ${ }^{\mathrm{c}}$, El-Shadan Tautolo ${ }^{\mathrm{c}}$, \\ Lindsay Plank ${ }^{\mathrm{e}}$, Shabnam Jalili-Moghaddam ${ }^{\mathrm{f}}$, Janis Paterson ${ }^{\mathrm{c}}$, Elaine Rush ${ }^{\mathrm{c}}$ \\ a School of Nursing, The University of Auckland, Auckland, New Zealand \\ b Department of Biostatistics and Epidemiology, Auckland University of Technology, Auckland, New Zealand \\ c Centre for Pacific Health and Development Research, Auckland University of Technology, Auckland, New Zealand \\ ${ }^{\mathrm{d}}$ Human Potential Centre, Auckland University of Technology, Auckland, New Zealand \\ e School of Medicine, the University of Auckland, Auckland, New Zealand \\ f Child Health Research Centre, Auckland University of Technology, Auckland, New Zealand
}

\section{A R T I C L E I N F O}

\section{Article history:}

Received 27 June 2016

Received in revised form 8 December 2016

Accepted 12 December 2016

Available online 29 December 2016

\section{Keywords:}

Physical activity

Measure

Accelerometry

Pacific health

Adolescents

\begin{abstract}
A B S T R A C T
This study aimed to assess the differences in participant retention and associations between physical activity and key variables when a range of accelerometer data inclusion criteria are employed. Data were drawn from 204 adolescents of Pacific Island heritage (survey, body composition, 7-day accelerometry) and their parents (date of birth, socioeconomic status) between October 2014 and February 2016 in Auckland, New Zealand. Data wear time criteria for inclusion were as follows: A) $>=10 \mathrm{~h} /$ weekday or $>=8 \mathrm{~h}$ weekend day, $>=5$ days (at least one weekend day); B) $>=10 \mathrm{~h} /$ weekday or $>=8 \mathrm{~h}$ weekend day, $>=4$ days; $\mathrm{C}$ ) $>=7 \mathrm{~h} /$ day, $>=3$ days; D) $>=10 \mathrm{~h} /$ day, $>=1$ day. Overall, $49 \%, 62 \%, 88 \%$, and $96 \%$ of participants met the criteria, respectively. Adjusted odds of meeting each criterion were examined using a multivariable logistic regression model. Almost $50 \%$ of participants were excluded by the most stringent inclusion criteria. Increased body fat percentage and proportion of time in moderate-to-vigorous activity were associated with decreased odds of meeting Criterions A and B. This research contributes to a growing understanding of the impact of differing accelerometer reduction approaches to sample retention and bias in adolescent physical activity research.
\end{abstract}

(c) 2017 The Authors. Published by Elsevier Inc. This is an open access article under the CC BY-NC-ND license (http://creativecommons.org/licenses/by-nc-nd/4.0/).

\section{Introduction}

Physical activity (PA) is fundamental to optimal health and development across the life course (World Health Organization, 2010). Objectively assessing the duration, intensity, and frequency of PA is essential for measuring the links between PA and health. Understanding these relationships can guide intervention development and evaluationand inform health promotion messages. Accelerometry is the method of choice to gain an unbiased, objective understanding of PA (Kelly et al., 2016). This technology facilitates the sensitive collection of movement data accumulated over specified epochs, enabling the calculation of time spent in differing activity intensities (e.g., moderate-tovigorous PA, MVPA).

Many questions still remain regarding accelerometer data treatment (e.g., minimum wear time for data inclusion) (Cain et al., 2013). Toftager et al. (2013)investigated the impact of differing accelerometer data treatment criteria on sample inclusion and bias in 1348 Danish adolescents aged 11-14 years. Adopting more stringent criteria resulted in significant reductions in participant inclusion. Moreover, participants

\footnotetext{
* Corresponding author.

E-mail address: melody.smith@auckland.ac.nz (M. Smith).
}

with larger body sizes and older adolescents were more likely to be excluded when more stringent criteria were employed. Reasons for accelerometer non-wear are multifactorial and can include individual factors such as response to peer influence (removal of unit because it is 'uncool') and social desirability bias (wearing units only when participating in PA) as well as researcher/measurement factors (e.g., researcher directives to remove individual participation in contact sports or water activities) (Kirby et al., 2012; Belton et al., 2013; Sirard and Slater, 2009). Accelerometer data treatment decisions can introduce unwanted bias and substantial loss of significant information, with impacts possibly greatest for those of importance in health research. This issue warrants further investigation to determine the most appropriate approach for a given population.

Pacific peoples are the fourth largest population group in New Zealand, comprising $7 \%$ of the total population. In 2014/2015, three times more Pacific children were classified obese (30\%) than the general population (Ministry of Health, 2015). Yet, Pacific youth are generally under-represented in PA and health research. The need to include youth from a range of sociodemographic groups in health research is recognised as important but challenging (e.g., due to logistical barriers such as transport or conflicting priorities, and implicit attitudinal barriers such as mistrust or differentiation) (Brannon et al., 2013). Study 
aims were (1) to quantify participant inclusion using a range of wear time criteria for accelerometer data reductionand (2) to calculate the odds of meeting wear time criteria across key variables, with a sample of New Zealand adolescents of Pacific Island heritage.

\section{Methods}

Data were drawn from the Pacific Islands Families cohort study of Pacific youth in Auckland, New Zealand (Rush et al., 2016). From October 2014 to February 2016, a subsample of the full cohort underwent body composition and metabolic risk assessment. Participants were stratified by sex and body weight decile at 11 years then purposively invited to participate (to facilitate representation across sex and weight). Informed consent was obtained from the individual and their parent. Participants were asked to fast the night before they were collected from their home and transported to Auckland Hospital where body composition was measured. Participants were fitted with an accelerometer on an elasticated belt and asked to wear the belt for waking hours over the next seven days except when bathing, swimming, sleeping, and playing contact sports. On the eighth day, the accelerometer was collected and koha (voucher) provided.

Ethical approval was provided by the Central Health and Disability Ethics Committee (14/CEN/108; 28 July 2014).

Factors hypothesised as being related to compliance with accelerometer wear instructions (and thus examined in the current study) are outlined below:

\subsection{PA and sedentariness}

\subsubsection{Accelerometry}

Activity was assessed using ActiGraph wGT3X + activity monitors (ActiGraph, Pensacola, FL). These small $(4.6 \mathrm{~cm} \times 3.3 \mathrm{~cm} \times 1.5 \mathrm{~cm})$ lightweight (19 g) units reliably measure motion across three axes using an electromechanical accelerometer (Lee et al., 2015). Units were initialised using a sampling frequency of $30 \mathrm{~Hz}$. After collection, raw data were aggregated into $30 \mathrm{~s}$ epochs using the low-frequency extension filter (Cain, 2013). Data were screened after every use to identify any obvious accelerometer malfunctions so these units could be removed from circulation. Epochs with more than 16,000 counts per minute were removed (Cain, 2013). Non-wear time was classified as $60 \mathrm{~min}$ or more of consecutive zero counts (Oliver et al., 2011). Re-wears (i.e., asking participants to wear the units for additional days if incomplete data were collected) were not logistically or economically feasible. Four combinations of criteria for inclusion in analyses were investigated: (A) at least 5 days (with at least one being a weekend day), with at least $10 \mathrm{~h}$ of valid data for weekdays and at least $8 \mathrm{~h}$ of valid data for weekend days (Cain, 2013); (B) at least 4 days, with at least $10 \mathrm{~h}$ of valid data for weekdays, or at least $8 \mathrm{~h}$ of valid data on weekend days; (C) at least 3 days with at least $7 \mathrm{~h}$ of valid data on each day (producing acceptable reliability in 5595 children aged 11 years $(R=0.7))$ (Mattocks et al., 2008); and (D) at least one day with at least $10 \mathrm{~h}$ of valid data (Tudor-Locke et al., 2010). Data were processed using ActiLife v6.11.9 (ActiGraph, Pensacola, FL). Proportion of time in MVPA was derived using the thresholds of Evenson et al. (2008).

\subsubsection{Self-reported sedentary behaviours}

Time spent in sedentary behaviours (e.g., watching TV) was assessed for weekdays and weekend days using items from the IPEN-A survey (International Physical Activity and the Environment Network (IPEN), 2013). Average time spent in sedentary behaviours on a usual day was calculated ((typical weekday minutes*5 + typical weekend day minutes*2) / 7).

\subsection{Social and demographic factors}

\subsubsection{Age and sex}

Date of birth (used to calculate age) and sex were collected from primary caregivers at baseline data collection (six weeks postpartum).

\subsubsection{Household socioeconomic status}

At the 14-year wave, primary caregivers were asked about their level of socioeconomic deprivation as measured by the New Zealand Index of socioeconomic Deprivation for Individuals (NZiDep) (Salmond et al., 2006). This deprivations-based measure of socioeconomic status asks about eight types of deprivation (e.g., "put up with feeling cold to save heating costs") with yes/no responses. The number of yes responses was summed and categorised into five bands.

\subsubsection{Response to peer influence}

Three items assessed the degree to which youth misbehaved to comply with peer pressure in the last year (e.g., "In the last year, how often have you done badly at something (e.g., schooolwork) just to please your friends?") (McDonough et al., 2015). A 5-point Likert scale response option was used, ranging from "never/almost never" to "always/almost always", and values summed to generate a scale of response to peer influence ( $1=$ not influenced; $15=$ heavily influenced).

\subsection{Body composition}

Total body fatness and abdominal fatness were measured using whole-body dual energy X-ray absorptiometry (DXA; model iDXA, software v.15; GE-Lunar, Madison, WI).

\subsection{Data analysis}

Descriptive statistics for the proportion of participants included were calculated for each criterion. Odds of meeting each of the four criteria were examined using a multivariable logistic regression model, adjusting for each sociodemographic and physical variable listed above. There were missing values in the peer pressure variable, which were accounted for by pooling the results from 50 complete datasets generated using multiple imputation.

\section{Results}

Of the 255 participants invited to participate, 205 agreed to be involved in the study. Reasons for non-participation were as follows: could not be contacted by phone, had moved out of Auckland, or refused. Of those included, one participant lost their accelerometer and another refused to wear the belt, yielding a final sample of 203. Table 1 outlines the characteristics of participants included in analyses. The proportion of participants included using criteria A-D were $49 \%, 62 \%$, $88 \%$, and $96 \%$, respectively. Table 2 shows the odds of meeting each criteria by key variables. Participants with higher body fatness were less likely to meet each inclusion criteria $A$ and $B$, as were participants with greater MVPA. No other evidence of associations was found.

\section{Discussion}

Accelerometer data treatment and reduction is notoriously challenging, and no best practice currently exists. This study aimed to inform data treatment approaches in adolescents of Pacific Island heritage.

Findings showed a substantial and increasing decline in the number of participants included when more stringent inclusion criteria were employed. Over half of the study participants were excluded using the IPEN-A criterion. This was much higher than the only other study to consider participant inclusion issues in adolescent PA research, where $15 \%$ of Danish adolescents were excluded using this criterion (Toftager 
Table 1

Participant characteristics.

\begin{tabular}{|c|c|c|c|c|c|c|}
\hline \multirow[b]{2}{*}{ Categorical measures } & \multicolumn{2}{|c|}{ Combined } & \multicolumn{2}{|c|}{$\begin{array}{l}\text { Females } \\
(n=100)\end{array}$} & \multicolumn{2}{|c|}{$\begin{array}{l}\text { Males } \\
(n=103)\end{array}$} \\
\hline & $n$ & $\%$ & $n$ & $\%$ & $n$ & $\%$ \\
\hline \multicolumn{7}{|l|}{ NZiDep deprivations } \\
\hline None & 26 & $13 \%$ & 10 & $10 \%$ & 16 & $16 \%$ \\
\hline One & 36 & $18 \%$ & 21 & $21 \%$ & 15 & $15 \%$ \\
\hline Two & 43 & $21 \%$ & 21 & $21 \%$ & 22 & $21 \%$ \\
\hline Three or four & 67 & $33 \%$ & 27 & $27 \%$ & 40 & $39 \%$ \\
\hline Five or more & 31 & $15 \%$ & 21 & $21 \%$ & 10 & $10 \%$ \\
\hline Continuous measures & Mean & SD & Mean & SD & Mean & SD \\
\hline Percentage body fat & 32.6 & 9.3 & 37.3 & 6.5 & 27.9 & 9.3 \\
\hline Peer pressure score ${ }^{c}$ & 2.7 & 2.6 & 2.5 & 2.4 & 2.9 & 2.8 \\
\hline Sedentary hours per day & 6.0 & 3.5 & 6.1 & 3.3 & 5.9 & 3.6 \\
\hline Percentage MVPA & 5.4 & 3.4 & 3.8 & 2.3 & 7.0 & 3.6 \\
\hline
\end{tabular}

Notes: MVPA = moderate-to-vigorous physical activity; NZiDep = New Zealand Index of Socioeconomic Deprivation for Individuals. Data collected in Auckland, New Zealand, between October 2014 and February 2016.

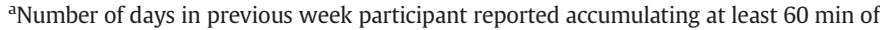
physical activity.

${ }^{\text {b}}$ Typical time (reported in minutes) spent outside school hours in sedentary behaviours.

${ }^{\mathrm{c}}$ Twenty-two missing values.

et al., 2013). Reasons for this difference are unclear; recalling the consistent findings regarding increased body size and reduced likelihood of meeting wear time criteria, this difference could be predominantly attributed to the high levels of overweight and obesity in the current study (71\% of boys and 76\% of girls) (Rush et al., 2016), compared with the Danish group (14.5\% and $14.6 \%$, respectively). This presents an interesting conundrum in that researchers need to consider the balance between participant inclusion and representation and PA measurement validity and reliability. Validity and reliability are fundamental drivers of decision making in research practice, while participant inclusion has received less attention. There have been increasing calls for strategies to encourage participant inclusion, particularly in those traditionally less represented in activity and behavioural research (Hindmarch et al., 2015).

Despite a number of strategies employed in this study, a substantial proportion of participants did not meet the data inclusion criteria. Additional approaches such as daily text messages (Belton et al., 2013) and provision of koha contingent on compliance (Sirard and Slater, 2009) may have improved wear times.

The odds of participant inclusion were then assessed for four wear time criteria using key characteristics hypothesised to influence participant compliance. Findings showed increased body fatness significantly decreased the odds of meeting the two most stringent wear time criteria, aligning with earlier adolescent research using body mass index (Toftager et al., 2013). In part, this may be due to other data processing decisions-for example, discerning between non-wear time and sedentary time is problematic. Higher sedentary time could be misclassified as non-wear time, resulting in the spurious removal of valid data (and reducing total wear time). To mitigate this somewhat, we chose a comparatively long non-wear time criteria to minimise this effect (Toftager et al., 2013). Irrespectively, strategies to encourage compliance in youth with greater body size are warrantedand could include using a clip rather than a belt for monitor attachment, personalising the device, and receiving feedback on activity levels (Kirby et al., 2012). The findings relating to MVPA are somewhat counter-intuitive. It is worth noting the low proportion of time spent in this activity intensity for this population (mean 5.4\%, SD 3.4\%). Again, it is possible this finding was due to other data processing decisions, as well as the metric applied for MVPA. For example, the absolute value of minutes in MVPA may have varied little between criteria, but the proportion of time spent in this variable would be expected to reducing significantly as the volume of data included increased. Taken another way, low wear time will likely have resulted in a greater proportion of the valid time being MVPA.

In contrast with earlier research, no other significant relationships were found, although there was a trend towards reduced odds of inclusion with increasing impact of peer pressure. It is possible that with a larger sample size, this relationship may have reached significance, and other relationships may have emerged.

Limitations are the small sample size and the focus on only one of the myriad data treatment decisions impacting activity outcomes derived from accelerometer data. Strengths include use of a more robust measure of body size than previous research, and including the novel aspect of the effect of peer pressure on compliance.

\section{Conclusion}

Application of more stringent wear time inclusion criteria resulted in declines in numbers of participants included in data analyses. Odds of meeting inclusion criteria were lower for those with higher body fatness, and for those with higher MVPA. Given an increased risk for exclusion bias and substantial data loss with the two most stringent criteria, the less stringent wear time criterion of Mattocks et al. (2008) appears an appropriate compromise in terms of participant inclusion and acceptable data reliability in this unique sample of Pacific adolescents.

\section{Conflict of interest statement}

The authors declare there are no conflicts of interest.

Table 2

Adjusted odds ratios for inclusion for each criterion.

\begin{tabular}{|c|c|c|c|c|c|c|c|c|c|}
\hline \multirow[b]{2}{*}{ Variable } & \multirow[b]{2}{*}{ Category or change } & \multicolumn{2}{|c|}{$\begin{array}{l}\geq 10 \text { h on weekdays, } \geq 8 \text { h } \\
\text { on weekend days, } \geq 5 \\
\text { valid days, including at } \\
\text { least one weekend day } \\
\end{array}$} & \multicolumn{2}{|c|}{$\begin{array}{l}\geq 10 \text { h on weekdays, } \geq 8 \mathrm{~h} \\
\text { on weekend days, } \geq 4 \\
\text { valid days }\end{array}$} & \multicolumn{2}{|c|}{$\begin{array}{l}\geq 7 \text { h on any day, } \geq 3 \\
\text { valid days (weekday or } \\
\text { weekend) } \\
\end{array}$} & \multicolumn{2}{|c|}{$\begin{array}{l}\geq 10 \text { h on any day, } \geq 1 \\
\text { valid days (weekday or } \\
\text { weekend) }\end{array}$} \\
\hline & & AOR & $95 \% \mathrm{CI}$ & AOR & $95 \% \mathrm{CI}$ & AOR & $95 \% \mathrm{CI}$ & AOR & $95 \% \mathrm{CI}$ \\
\hline \multirow{2}{*}{ Sex } & Female & 1.000 & (Reference) & 1.000 & (Reference) & 1.000 & (Reference) & 1.000 & (Reference) \\
\hline & Male & 0.508 & $(0.24,1.07)$ & 0.578 & $(0.27,1.23)$ & 0.993 & $(0.33,2.98)$ & 1.280 & $(0.18,9.05)$ \\
\hline NZiDep & Per deprivation & 0.979 & $(0.83,1.16)$ & 0.976 & $(0.82,1.16)$ & 0.911 & $(0.71,1.17)$ & 1.153 & $(0.73,1.81)$ \\
\hline PC body fat & Per unit increase & 0.950 & $(0.92,0.99)^{* *}$ & 0.962 & $(0.93,1.00)^{*}$ & 0.947 & $(0.90,1.00)$ & 0.965 & $(0.88,1.06)$ \\
\hline Peer pressure score & Per unit increase & 0.910 & $(0.80,1.04)$ & 0.889 & $(0.78,1.01)$ & 0.853 & $(0.71,1.03)$ & 0.777 & $(0.54,1.11)$ \\
\hline Sedentary hours & Per additional hour & 1.001 & $(0.92,1.09)$ & 1.072 & $(0.98,1.17)$ & 1.037 & $(0.91,1.18)$ & 1.079 & $(0.86,1.36)$ \\
\hline \%MVPA & Per unit increase & 0.897 & $(0.81,1.00)^{*}$ & 0.887 & $(0.80,0.98)^{*}$ & 0.986 & $(0.85,1.14)$ & 1.017 & $(0.78,1.32)$ \\
\hline
\end{tabular}

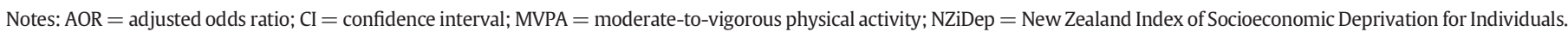
Data collected in Auckland, New Zealand, between October 2014 and February 2016.

* $p<0.05$.

** $p<0.01$ 


\section{Acknowledgements}

This study was funded by the Health Research Council of New Zealand (grant number 14-156). We wish to sincerely thank Des Ryan for his substantial contribution to this study. We gratefully acknowledge the significant and ongoing involvement of families participating in the Pacific Islands Families study.

\section{References}

Belton, S., O'Brien, W., Wickel, E.E., Issartel, J., 2013. Patterns of noncompliance in adolescent field-based accelerometer research. J. Phys. Act. Health 10, 1181-1185.

Brannon, E.E., Kuhl, E.S., Boles, R.E., et al., 2013. Strategies for recruitment and retention of families from low-income, ethnic minority backgrounds in a longitudinal study of caregiver feeding and child weight. Child Health Care 42 (3), 198-213.

Cain, K., 2013. IPEN Adolescent Accelerometer Scoring Protocol. [cited 20151 August] Available from:. http://www.ipenproject.org/methods_accelerometers.html.

Cain, K.L., Sallis, J.F., Conway, T.L., Van Dyck, D., Calhoon, L., 2013. Using accelerometers in youth physical activity studies: a review of methods. J. Phys. Act. Health 10 (3), 437-450.

Evenson, K.R., Catellier, D.J., Gill, K., Ondrak, K.S., McMurray, R.G., 2008. Calibration of two objective measures of physical activity for children. J. Sports Sci. 26 (14), 1557-1565.

Hindmarch, P. Hawkins, A., McColl, E., et al., 2015. Recruitment and retention strategies and the examination of attrition bias in a randomised controlled trial in children's centres serving families in disadvantaged areas of England. Trials 16, 79.

International Physical Activity and the Environment Network (IPEN), 2013k. IPEN Adolescent Survey for Children-English. San Diego State University, San Diego, USA.

Kelly, P., Fitzsimons, C., Baker, G., 2016. Should we reframe how we think about physical activity and sedentary behaviour measurement? Validity and reliability reconsidered. Int. J. Behav. Nutr. Phys. Act. 13, 32.
Kirby, J., Tibbins, C., Callens, C., et al., 2012. Young people's views on accelerometer use in physical activity research: findings from a user involvement investigation. ISRN Obes. 948504.

Lee, J.A., Williams, S.M., Brown, D.D., Laurson, K.R., 2015. Concurrent validation of the ActiGraph gt $3 \mathrm{x}+$, Polar Active accelerometer, Omron HJ-720 and Yamax Digiwalker SW-701 pedometer step counts in lab-based and free-living settings. J. Sports Sci. 33 (10), 991-1000.

Mattocks, C. Ness, A., Leary, S., et al., 2008. Use of accelerometers in a large field-based study of children: protocols, design issues, and effects on precision. J. Phys. Act. Health 5 (Suppl. 1), S98-S111.

McDonough, M.H., Jose, P.E., Stuart, J., 2015. Bi-directional effects of peer relationships and adolescent substance use: a longitudinal study. J. Youth Adolesc.

Ministry of Health, 2015. 'Ala Mo'ui Progress Report. Author, Wellington, New Zealand.

Oliver, M., Badland, H.M., Schofield, G.M., Shepherd, J., 2011. Identification of accelerometer non-wear time and sedentary behavior. Res. Q. Exerc. Sport 82 (4), 593-597.

Rush, E., Oliver, M., Plank, L.D., et al., 2016. Cohort profile: Pacific Islands Families (PIF) growth study Auckland, New Zealand. BMJ Open 6 (11), e013407.

Salmond, C., Crampton, P., King, P., Waldegrave, C., 2006. NZiDep: a New Zealand index of socioeconomic deprivation for individuals. Soc. Sci. Med. 62 (6), 1474-1485.

Sirard, J.R., Slater, M.E., 2009. Compliance with wearing physical activity accelerometers in high school students. J. Phys. Act. Health 6 (Suppl. 1), S148-S155.

Toftager, M. Kristensen, P.L., Oliver, M., et al., 2013. Accelerometer data reduction in adolescents: effects on sample retention and bias. Int. J. Behav. Nutr. Phys. Act. 10, 140.

Tudor-Locke, C., Brashear, M.M., Johnson, W.D., Katzmarzyk, P.T., 2010. Accelerometer profiles of physical activity and inactivity in normal weight, overweight, and obese U.S. men and women. Int. J. Behav. Nutr. Phys. Act. 7 (1).

World Health Organization, 2010. Global Recommendations on Physical Activity for Health. Author, Geneva. 